\title{
Tritrichomonas foetus: a scanning electron microscopy study of erythrocyte adhesion associated with hemolytic activity
}

\author{
Geraldo Attilio De CARLI ${ }^{a *}$, Tiana TASCA ${ }^{\mathrm{a}, \mathrm{b}}$, Fernanda PIRES BorGES ${ }^{\mathrm{a}}$

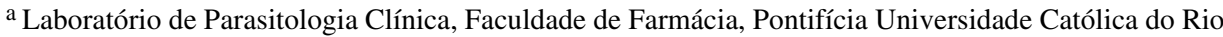 \\ Grande do Sul, Av. Ipiranga 6681, 90619-900 Porto Alegre, RS, Brazil \\ b Departamento de Bioquímica, Instituto de Ciências Básicas da Saúde, Universidade Federal do Rio \\ Grande do Sul, Rua Ramiro Barcelos 2600 - Anexo. 90035-003 Porto Alegre, RS, Brazil
}

(Received 3 July 2003; accepted 21 August 2003)

\begin{abstract}
The in vitro hemolytic activity of Tritrichomonas foetus was investigated. The parasite was tested against human erythrocytes of groups $\mathrm{A}, \mathrm{B}, \mathrm{AB}$, and $\mathrm{O}$, and against erythrocytes of nine adult animals of different species (the rabbit, rat, chicken, cat, dog, swine, horse, bovine, and sheep). The results showed that $T$. foetus strains (ATCC KV1, K, PAL, 5022, RJ, 90) did not present any hemolytic activity against any human erythrocyte group nor against rabbit, rat, chicken, cat, dog and swine erythrocytes. T. foetus strains, however, lysed horse, bovine, and sheep erythrocytes. No hemolysin released by the parasites could be identified. Hemolysis did not occur with trichomonad culture supernatants, with sonicated extracts of $T$. foetus, nor with killed organisms. Scanning electron microscopy (SEM) showed that human erythrocytes did not adhere to the trophozoites, in contrast horse erythrocytes adhered to the surface of the parasites and were phagocytosed for up to 90 min. The parasites are able to exert their cytopathic effects through: (a) physical contact established between the two cell surfaces, (b) toxins released from parasites into the interaction media, or (c) the association of both mechanisms. Further studies are necessary to clarify the importance of the hemolytic activity in the biology of $T$. foetus.
\end{abstract}

Tritrichomonas foetus / hemolytic activity / scanning electron microscopy / erythrophagocytosis / cytopathic effects

\section{INTRODUCTION}

Tritrichomonas foetus is responsible for bovine urogenital trichomoniasis. It is a venereal disease transmitted by coitus as well as by sub-standard artificial insemination. The protozoan is observed in the urogenital cavity of cows and it is able to migrate upward through the cervix and invade the uterus [17]. In bulls, the most common site of infection is the preputial cavity, although the epididymis, and semi- nal vesicles may sometimes be involved [2, 17, 21]. Among infected calves, the occurrence of endometritis, accompanied by uterine, cervical, and vaginal catarrh, is frequent. Since endometritis and uterine catarrh may prevent fertilisation affecting the regularity of the estrous cycle, some of the infected calves may remain permanently sterile [2, 22].

The interaction of $T$. foetus with the epithelium lining of the urogenital cavities

* Corresponding author: gdecarli@ portoweb.com.br 
is the initial and crucial step for the establishment of bovine trichomoniasis [22]. Although extensively studied, the mechanisms of the pathogenicity of $T$. foetus have not yet been well defined. The pathogenicity of $T$. foetus has been previously reported, employing growth characteristics [20], mouse inoculation [13] and in vitro experiments using epithelial cells to investigate pathogenic trichomonad-host cell interaction relationships $[31,32]$. The hemolytic activities of different species of Trichomonadida, including Trichomonas vaginalis $[6,7,16,19,27], T$. gallinae $[10$, 11, 33] Tritrichomonas foetus [8, 9] and Tritrichomonas suis [8], have been studied using in vitro methods.

The investigation of hemolytic activity constitutes an experimental model, using red blood cells as the target membranes, to better understand the cytopathic mechanism used by $T$. foetus. The aim of this study was to determine the hemolytic activity of six strains of $T$. foetus and to investigate the contact-dependent mechanism between the parasites and the different erythrocytes (fresh human blood and different adult animal species) by scanning electron microscopy (SEM).

\section{MATERIALS AND METHODS}

\subsection{Parasite culture conditions}

Six $T$. foetus strains used in this study (ATCC KV1, K, PAL, 5022, RJ, 90) were obtained from Professors Wanderley de Souza, Marlene Benchimol and Fernando C. Silva Filho (UFRJ, Instituto de Biofísica Carlos Chagas Filho, Rio de Janeiro, Brazil) and Dr Helio Guida (EMBRAPA, Seropédica, Rio de Janeiro, Brazil). The trichomonads were cultured axenically in trypticase-yeast extract-maltose (TYM) medium [12], supplemented with $10 \%$ heat inactivated bovine serum, penicillin (1000 IU/ $\mathrm{mL})$ and streptomycin sulphate $(1 \mathrm{mg} / \mathrm{mL})$ at $37^{\circ} \mathrm{C}$. The $\mathrm{pH}$ of the TYM medium was adjusted to 7.2 . The strains were kept alive in liquid nitrogen $\left(-196^{\circ} \mathrm{C}\right)$ with dimethyl sulfoxide (DMSO) [25]. The trichomonads were in the logarithmic phase of growth and subcultured every $48 \mathrm{~h}$ and exhibited more than $95 \%$ mobility and normal morphology. The protozoa were counted with a hemocytometer and adjusted to a concentration of $1 \times 10^{6}$ cells $/ \mathrm{mL}$ in TYM medium.

\subsection{Erythrocytes}

Fresh human blood was obtained at the City's Emergency Hospital's Blood Center and also from volunteer donors, and fresh animal blood was obtained from nine different adult animal species (rabbit, rat, chicken, cat, dog, swine, horse, bovine, and sheep). The blood was diluted in an equal volume of Alsever's solution. The plasma was discarded by centrifugation $(250 \times g$ for $5 \mathrm{~min}$ ) and the erythrocytes were washed three times in sterile phosphate saline buffer (PBS). Each experiment was performed using fresh erythrocytes from all human blood groups and adult animals. Whole human blood samples were previously examined, and determined to be hepatitis $\mathrm{B}$ antigen ( $\mathrm{HBsAg}$ ) negative, and human immunodeficient virus (HIV-antibody) negative.

\subsection{Hemolysis assay}

The trophozoites were harvested from a $24 \mathrm{~h}$ culture (viability > 95\%) in TYM medium, and washed three times in TYM medium by centrifugation $(750 \times g$ for $10 \mathrm{~min}$ ). A volume of $50 \mu \mathrm{L}$ of washed fresh undiluted erythrocytes was mixed with $2.5 \mathrm{~mL}$ of TYM medium containing a total of $1 \times 10^{6}$ trophozoites of $T$. foetus. After $18 \mathrm{~h}$ of incubation at $37^{\circ} \mathrm{C}$, the mixture was centrifuged $(250 \times g$ for $10 \mathrm{~min})$. Absorbance of the supernatants and controls were measured at $540 \mathrm{~nm}$. Control tubes were included in all assays and the spontaneous hemolysis was also controlled. The results were expressed as percentages of 
total hemolysis $(100 \%)$. The mean and the standard error of the hemolytic activity of trichomonads with the different erythrocytes were calculated after performing the assay at least four times, each in triplicate. In order to investigate the possible presence of a hemolysine or a metabolism product responsible for the hemolysis, the assay was performed with culture supernatants from 24 and $48 \mathrm{~h}$, hemolysis supernatant, parasite sonicated extracts and killed $T$. foetus instead of the trichomonads. The parasites were sonicated in the late exponential phase and washed three times in PBS, $\mathrm{pH} 7.2$, by centrifugation at $2000 \mathrm{rpm}$. The washed cells were resuspended at a density of $1 \times$ $10^{6}$ organisms/mL in PBS, $\mathrm{pH} 7.2$, then were subjected to five cycles of sonication for $10 \mathrm{~s}$ at 50 watts in an ice bath. The samples were then centrifuged and the supernatants were filtered through a $0.22 \mu \mathrm{m}$ filter membrane (Millipore) in order to perform the hemolysis assays. Killed T. foetus was obtained by freezing. Statistical analysis was conducted by one-way ANOVA (Analysis of Variance) followed by the Duncan post-hoc test, considering a level of significance of $5 \%$.

\subsection{Scanning electron microscopy}

In order to investigate the interaction between $T$. foetus and human $\mathrm{O}$ group erythrocytes or horse erythrocytes, the cells were collected by centrifugation at 6000 $\times g$ for $5 \mathrm{~min}$ and allowed to interact for 30 , 60 and 90 min in PBS using an erythrocyte: protozoan ratio of 100:1. After interaction, the cells were fixed for $2 \mathrm{~h} 30 \mathrm{~min}$ at room temperature in a solution containing $2.5 \%$ glutaraldehyde in $0.1 \mathrm{M}$ cacodylate buffer $(\mathrm{pH}$ 7.2). The cells were then allowed to adhere to glass coverslips previously coated with $0.1 \%$ poly-L-lysine (Sigma, St. Louis, MO, USA) and post-fixed for $2 \mathrm{~h}$ with $1 \% \mathrm{OsO}_{4}$. The fixed samples were dehydrated in ethanol, critically point dried with a Bal-Tec CPD 030 critical-point dryer and lightly coated with gold particles in a Bal-Tec SCD 005 sputter coater [11, 29,
34]. Observations were carried out using a Philips XL30 scanning electron microscope (SEM).

\section{RESULTS}

The strains of Tritrichomonas foetus (ATCC KV1, K, PAL, 5022, RJ, 90) did not present any hemolytic activity against any of the human blood group erythrocytes, nor against rabbit, rat, chicken, cat, dog and swine erythrocytes (data not shown). In contrast, high hemolytic activity, ranging from $31 \pm 1.3$ to $96 \pm 0.9 \%$ (mean \pm S.E.), was detected when $T$. foetus strains were tested against horse, bovine, and sheep erythrocytes (Fig. 1). Hemolytic activity was maintained after a serial transfer in axenic culture for six months. The $T$. foetus trophozoites observed at the end of the hemolysis assays were alive and were successfully cultured in TYM medium. The cellular viability was not affected by experimental conditions.

No hemolysin released by the parasites could be identified. Hemolysis did not occur with trichomonad culture supernatants at 24 and $48 \mathrm{~h}$. Hemolytic activity was not observed with the hemolysis supernatant obtained at $18 \mathrm{~h}$, not with the sonicated extracts of trichomonads, nor with previously killed $T$. foetus (data not shown).

The SEM showed that human O group erythrocytes do not adhere to the parasite (Figs. 2-5). In contrast, SEM demonstrated that horse erythrocytes adhered to the trophozoites of $T$. foetus, developed an amoeboid morphology, and crawled over and under the erythrocytes. Figures 6 to 8 show the contact between the protozoan and horse erythrocytes after $30 \mathrm{~min}$. Figure 7 shows the amoeboid morphology of the trophozoites in contact with the horse erythrocytes. After just $30 \mathrm{~min}$, strong adhesion between both cells was observed (Fig. 8). Figures 9 to 11 show the adhesion between protozoan and horse erythrocytes after 60 min, and Figures 12 and 13 demonstrate adhesion after $90 \mathrm{~min}$. All of these figures depict a suction mechanism that strongly 
$\square$ Bovine $\square$ Horse $\square$ Sheep

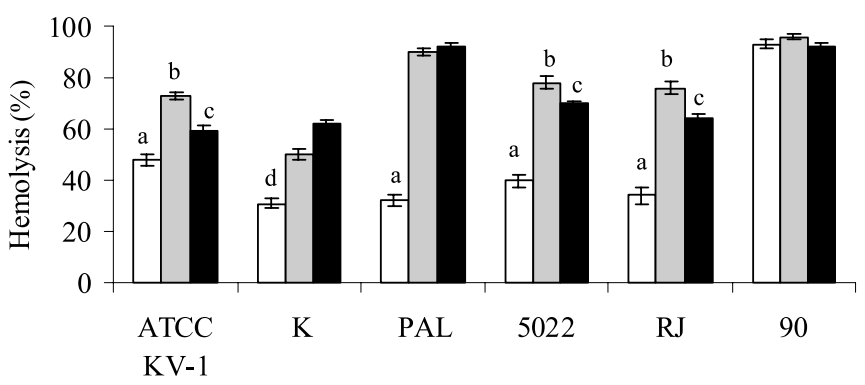

Tritrichomonas foetus strains

Figure 1. Hemolytic activity of Tritrichomonas foetus strains against bovine, horse and sheep erythrocytes. Experimental conditions are described in Materials and methods. Bars represent means \pm S.E. of four different experiments using different cell suspensions, each in triplicate. The results were analysed statistically by one-way ANOVA, followed by the Duncan post-hoc test: (a) significant difference from horse and sheep erythrocytes $(P<0.05)$; (b) significant difference from bovine and sheep erythrocytes $(P<0.05)$; (c) significant difference from bovine and horse erythrocytes $(P<0.05)$; (d) significant difference from sheep erythrocytes $(P<0.05)$.
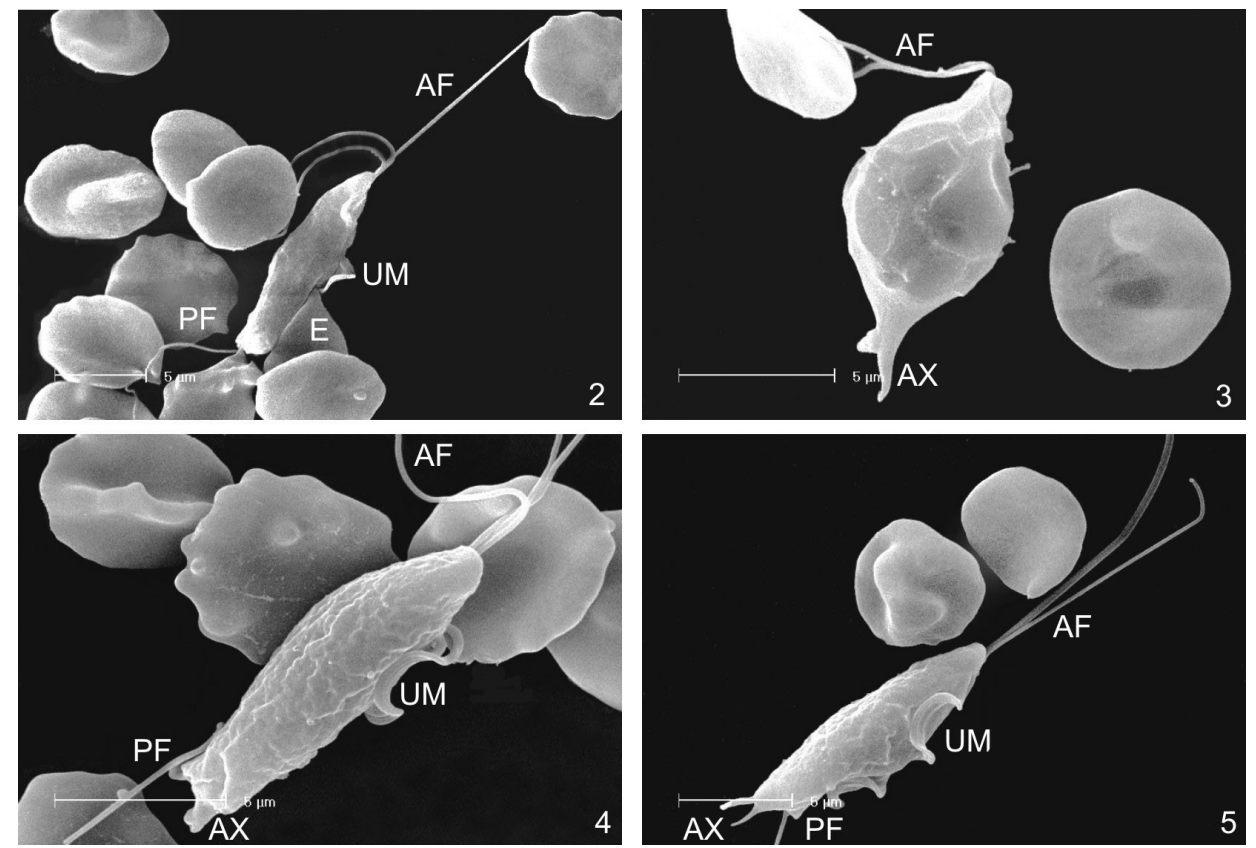

Figures 2-5. Interaction between Tritrichomonas foetus and human erythrocytes. Figures 2 and 3 show the contact between the K strain and erythrocytes after 30 and $60 \mathrm{~min}$, respectively. Figures 4 and 5 show the adhesion between both cells after 90 min. (E: erythrocyte, AF: anterior flagella, AX: axostyle, UM: undulating membrane, PF: posterior flagellum.) 

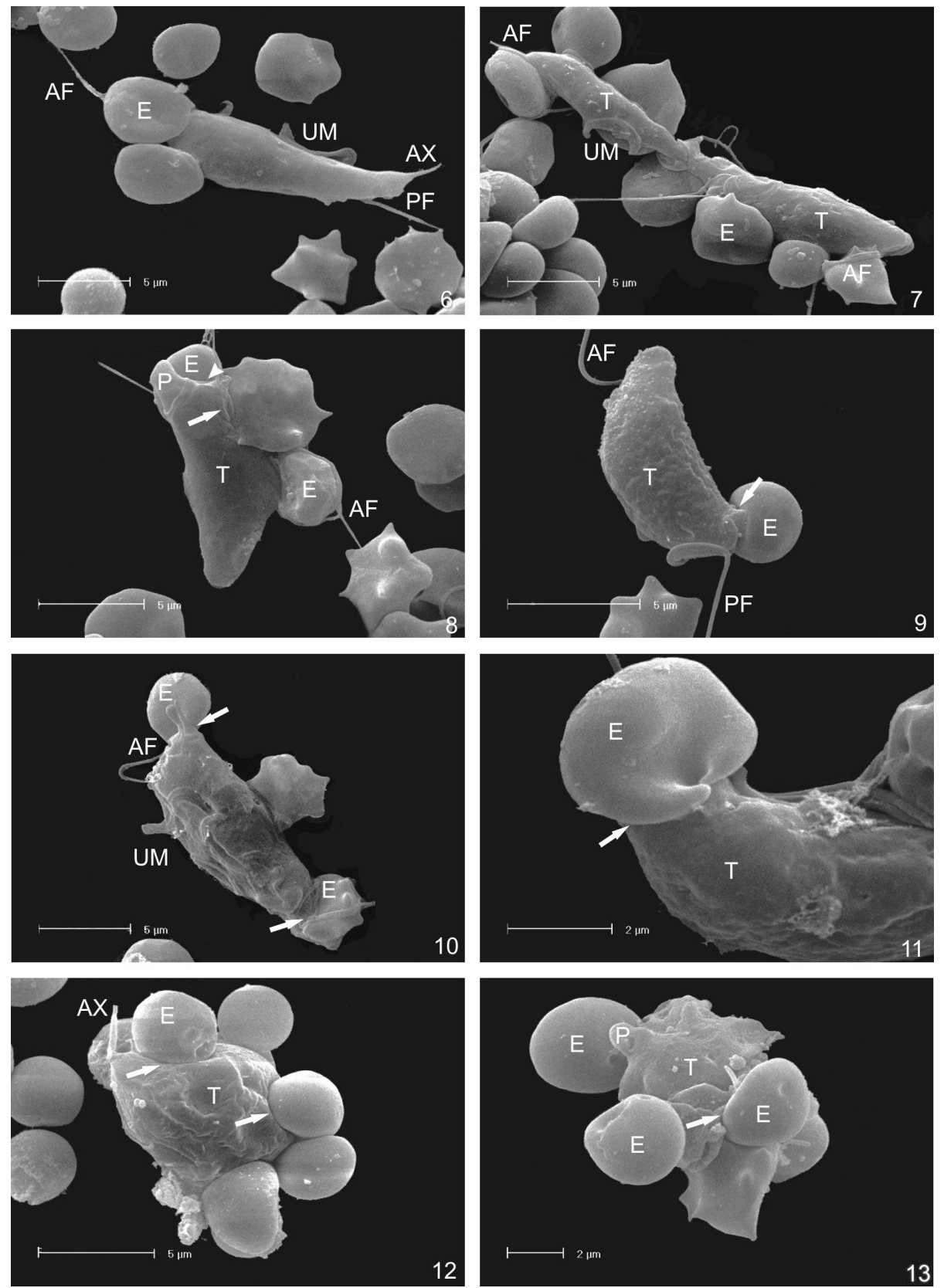

Figures 6-13. Interaction between Tritrichomonas foetus and horse erythrocytes. Figures 6 to 8 show the contact between the K strain and erythrocytes after 30 min. Figures 9 to 11 show the interaction between both cells after 60 min of incubation and Figures 12 and 13, after 90 min. (E: erythrocyte, T: trichomonad, AF: anterior flagella, AX: axostyle, UM: undulating membrane, PF: posterior flagellum, P: pseudopods). Note the "suction" mechanism of erythrophagocytosis (arrows) and the internalisation of the erythrocyte on the surface of the trichomonad (Fig. 8, arrowhead). 
suggests erythrophagocytosis developed by the parasite. In addition, Figure 10 shows the involvement of the anterior and posterior flagella, and even the axostyle, probably in the capture of the erythrocyte. In addition pseudopods were also observed (Figs. 8 and 13). The horse erythrocytes adhered to the protozoan body and were phagocytosed over the entire flagellate surface.

\section{DISCUSSION}

In the current report, in vitro studies were conducted to provide more information regarding the direct interaction of $T$. foetus and erythrocytes. Hemolytic activity has been demonstrated in several protozoan parasites such as Trypanosoma congolense [36], T. brucei [37] and Entamoeba histolytica [23]. The hemolytic activity of $T$. vaginalis and the correlation with the virulence of the strains has been previously described [7-9, 16, 19]. The mechanism of hemolysis is not yet well established and may be different for each species. T. congolense hemolysis has been related to fatty acid release from endogenous phosphatidyl choline by a phospholipase A [35].

Our data suggest that the adhesiveness, amoeboid morphology, and motility of $T$. foetus may be the consequence of specific recognition on the surface of parasites and may play an important role in the mechanisms of hemolysis in $T$. foetus. When horse erythrocytes were maintained with the trophozoites, the SEM demonstrated erythrophagocytosis by the parasites, suggesting a contact-dependent mechanism of the hemolytic activity. Rendón-Maldonado et al. [28] have observed two forms of erythrophagocytosis by $T$. vaginalis. The first involves a suction mechanism, with the elongation of the erythrocyte plasma membrane and internalisation through a narrow endocytic channel, while the second proceedes through the emission of a phagocytic stoma.

In addition, Fiori et al. [14] also proposed a mechanism to explain that the hemolytic activity of $T$. vaginalis was a contact and temperature dependent phenomenon and hypothesised that cytopathic effects could be related to pore-forming in the membrane of erythrocytes. Multiple activities of cysteine proteinases $(\mathrm{CP})$ have been found among parasitic protozoa [26], including T. foetus [24]. However, the CP in $T$. foetus are associated with the cytotoxicity exerted by the parasite to cultured epithelial cells rather than cytoadhesion [31]. In addition, $\mathrm{CP}$ inhibitors greatly reduce erythrocyte lysis, suggesting that $\mathrm{CP}$ may be a lytic factor involved in hemolysis [6]. Furthermore, studies focusing on the trichomonad-cell interaction have pointed out that the parasites can exert their cytopathic effects through: (a) physical contact established between the two cell surfaces, (b) toxins released from parasites into the interaction media, or (c) the association of both mechanisms [5].

Here we suggest that $T$. foetus hemolysis, like other cell-cell interaction processes, is a very complex phenomenon which is always preceded by cellular recognition. The presence of pseudopods in T. foetus (Figs. 8 and 13) may represent a very efficient mechanism for the parasite to make contact with the erythrocyte surface. Furthermore, the movement of the four flagella found in T. foetus is of importance not only to the locomotion of the parasite into liquid media, but also to the induction of liquid currents that become parasites able to easily uptake nutrients from the surrounding medium [20]. Likewise, Figure 10 shows the anterior and posterior flagella, and even the axostyle, probably involved in the capture of the erythrocyte.

Da Silva et al. [5] showed strong agglutination between $T$. foetus and human A group erythrocytes, suggesting the occurrence of an adhesin associated with the parasite surface. Indeed, the authors used the same K strain and human erythrocytes that we used in the present study and observed an increasing intensity of adhesion for up to $30 \mathrm{~min}$ of incubation. In contrast, we did not observe adhesion between 
human erythrocytes and the trophozoites of $T$. foetus for up to $90 \mathrm{~min}$. To explain these results, we suggest that the erythrocytes from different donors could express different surface molecules which interact with the parasites through divergent pathways resulting, or not, in adhesion.

In addition, lectin molecules found at the cell surface of trichomonads appear to be important for epithelial cell recognition by these organisms [1]. Bonilha et al. [3] showed that sialic acid residues present on the surface of Chinese hamster ovary (CHO) cells may favour the cytoadhesion of $T$. foetus, whilst hampering that of T. vaginalis. These findings strongly suggest an important role for host-cell surface glycoconjugates during the cytoadhesion of trichomonads, demonstrating that the recognition of the erythrocytes by $T$. foetus may be partially related to some parasitic surface lectins. Furthermore, a $118 \mathrm{kDa}$ surface molecule enables $T$. foetus to recognise laminin-1, an extracellular matrix (ECM) glycoprotein, and to adhere to lamininrevested substrates [32]. The parasite is also able to recognise fibronectin, another ECM glycoprotein, through some manose residue-containing glycoconjugates [30]. Lectin molecules and proteins that recognise laminin and fibronectin may play an important role in the process by which the type of erythrocyte is recognised and adhered to by T. foetus.

Our results showed that, for T. foetus, no hemolytic activity could be detected in culture supernatants, suggesting that hemolysis was not dependent upon the release of hemolysin or soluble metabolites by the parasite. Other authors $[4,18]$ have shown that all isolates of $T$. foetus tested by them presented a hemolysin. Differences in the experimental conditions, such as $\mathrm{pH}$ values and temperatures could influence the identification of these hemolysins [15]. In addition, it is possible that cysteine proteinases identified in $T$. foetus [24] could inactivate a released hemolysin of the parasite, which could be studied by the addition of protei- nase inhibitors. Further studies are necessary to clarify the importance of the hemolytic activity in the biology of $T$. foetus.

\section{ACKNOWLEDGEMENTS}

To Dr. Sérgio De Meda Lamb for support and to Rafael V. Michel for technical assistance. To Centro de Microscopia e Microanálises (CEMM - PUCRS) for SEM technical assistance. This work was partially supported by grants from Conselho Nacional de Desenvolvimento Científico e Tecnológico (CNPq) \# 470148/01-2, Fundação de Amparo à Pesquisa do Estado do Rio Grande do Sul (FAPERGS), and Faculdade de Farmácia, PUCRS. T.T. is recipient of a $\mathrm{CNPq}$ fellowship.

\section{REFERENCES}

[1] Babál P., Russel L.C., Sialic acid-specific lectin mediated adhesion of Tritrichomonas foetus and Tritrichomonas mobiliensis, J. Parasitol. 85 (1999) 33-40.

[2] BonDurant R.H., Honigberg B.M., Trichomonads of veterinary importance, in Kreier J. (Ed.), Parasitic Protozoa, Academic Press, New York, 1994, pp. 111-206.

[3] Bonilha V.L., Ciavaglia M.C., de Souza W. Silva Filho F.C., The involvement of terminal carbohydrates of the mammalian cell surface in the cytoadhesion of trichomonads, Parasitol. Res. 81 (1995) 121-126.

[4] Burgess D.E., Knoblock K.F., Daugherty T., Robertson N.P., Cytotoxic and hemolytic effects of Tritrichomonas foetus on mammalian cells, Infect. Immun. 58 (1990) 36273632.

[5] da Silva N.S., Dias Filho B.P., de Souza W., Structural changes at the site of Tritrichomonas foetus-erythrocyte interaction, Cell Struct. Funct. 21 (1996) 245-250.

[6] Dailey D.C., Chang T., Alderete J.F., Characterization of Trichomonas vaginalis haemolysis, Parasitology 101 (1990) 171-175.

[7] De Carli G.A., Brasseur P., Savel J., Activité hémolitique de différentes souches et clones de T. vaginalis, Bull. Soc. Fr. Parasitol. 7 (1989) 13-16.

[8] De Carli G.A., Brasseur P., Rott M., da Silva A.C.A., Wendorff A., Benchimol M., Determination of hemolytic activity of different strains of trichomonads of genus Trichomonas Donné, 1836 and Tritrichomonas Kofoid, 1920, J. Protozool. Res. 4 (1994) 158-163.

[9] De Carli G.A., Brasseur P., da Silva A.C., Wendorff A., Rott M., Hemolytic activity of 
Trichomonas vaginalis and Tritrichomonas foetus, Mem. Inst. Oswaldo Cruz 97 (1996) 107-110.

[10] De Carli G.A., da Silva A.C., Wendorff A., Rott M., Lysis of erythrocytes by Trichomonas gallinae, Avian Dis. 40 (1996) 228-230.

[11] De Carli G.A., Tasca T., Trichomonas gallinae: a possible contact-dependent mechanism in the hemolytic activity, Vet. Parasitol. 87 (2002) 111-118.

[12] Diamond L.S., The establishment of various Trichomonas of animals and man in axenic cultures, J. Parasitol. 43 (1957) 488-490.

[13] Dohnalová M., Kulda J., Pathogenicity of Tritrichomonas foetus for the laboratory mouse, J. Parasitol. A 22 (1975) 61.

[14] Fiori P.L., Rappelli P., Rocchigiani A.M., Cappuccinelli P., Trichomonas vaginalis haemolysis: Evidence of functional pores formation on red cell membranes, FEMS Microbiol. Lett. 109 (1993) 13-18.

[15] Garber G.E., Lemchuck-Favel L.T., Characterization and purification of extracellular proteases of Trichomonas vaginalis, Can. J. Microbiol. 35 (1989) 903-909.

[16] Grys E.A., Hernik A., Hemolysis of human and rabbit erythrocytes by $T$. vaginalis, Wiad. Parazytol. 19 (1973) 399-400.

[17] Honigberg B.M., Trichomonads of veterinary importance, in: Kreier J.P. (Ed.), Parasitic Protozoa, Academic Press, New York, 1978, pp. 163-273.

[18] Kennett M.J., Hook R.R., Tritrichomonas foetus: characterization of isolates and partial purification of a secreted cytotoxin, Exp. Parasitol. 102 (2002) 1-8.

[19] Krieger J.N., Poisson M.A., Rein M.F., Betahemolytic activity of Trichomonas vaginalis correlates with virulence, Infect. Immun. 41 (1983) 1291-1295.

[20] Kulda J., Honigberg B.M., Behavior and pathogenicity of Tritrichomonas foetus in chick liver cell cultures, J. Parasitol. 16 (1969) 479495.

[21] Levine N.D., Protozoan parasites of domestic animal and of man, Burgess Publishing Co., Minneapolis, 1973.

[22] López L.B., Braga M.B.M., López J.O., Arroyo R., Silva Filho F.C., Strategies by which some pathogenic trichomonads integrate diverse signals in the decision-making process, An. Acad. Bras. Cienc. 72 (2000) 173-186.

[23] Lopez-Revilla R., Said-Fernandez S., Cytopathogenicity of Entamoeba histolytica hemolytic activity of trophozoite homogenates, Am. J. Trop. Med. Hyg. 29 (1980) 200-212.

[24] Mallinson D.J., Livingstone J., Appleton K.M., Less S.J., Coombs G.H., North M., Multiple cysteine proteinases of the patho- genic protozoan Tritrichomonas foetus: identification of seven diverse and differentially expressed genes, Microbiology 141 (1995) 3077-3085.

[25] McMillan A., Laboratory Diagnostic Methods and Cryopreservation of Trichomonads, in: Honigberg B.M. (Ed.), Trichomonads Parasitic in Humans, Springer-Verlag, New York, 1990, pp. 297-310.

[26] North M.J., Mottram J.C., Coombs G.H., Cystein proteinases of parasitic protozoa, Parasitol. Today 6 (1990) 270-275.

[27] Potamianos S., Mason P.R., Read J.S., Chikungauwo S., Lysis of erythrocytes by Trichomonas vaginalis, Biosci. Rep. 12 (1992) 387-385.

[28] Rendón-Maldonado J.G., Espinosa-Cantellano G.R.A, Martinez-Palomo A., Trichomonas vaginalis: In vitro phagocytosis of lactobacilli, vaginal epithelial cells, leukocytes, and erythrocytes, Exp. Parasitol. 89 (1998) 241-250.

[29] Rosset I., Tasca T., Tessele P.M., De Carli G.A., Scanning electron microscopy on the investigation of in vitro hemolytic activity of Trichomonas vaginalis, Parasitol. Res. 88 (2002) 356-359.

[30] Silva Filho F.C., Elias C.A., de Souza W., Role of divalent cations, $\mathrm{pH}$, cytoskeleton components and surface charge on the adhesion of Trichomonas vaginalis to a polystyrene substrate, Mem. Inst. Oswaldo Cruz 82 (1987) 379-384.

[31] Silva Filho F.C., de Souza W., The interaction of Trichomonas vaginalis and Tritrichomonas foetus with epithelial cells in vitro, Cell Struct. Funct. 13 (1988) 301-310.

[32] Silva Filho F.C., de Souza W., Lopes J.D., Presence of laminin-binding proteins in trichomonads and their role in adhesion, Proc. Natl. Acad. Sci. USA 85 (1988) 8042-8046.

[33] Tasca T., De Carli G.A., Hemolytic activity of fresh isolates and clones of Trichomonas gallinae, Parasitol. al Día 23 (1999) 69-73.

[34] Tasca T., De Carli G.A., Growth kinetic study of Tetratrichomonas didelphidis isolated from opossum Lutreolina crassicaudata and association with a prokaryotic cell, Parasitol. Res. 87 (2001) 626-630.

[35] Tizard I.R., Holmes W.L., The generation of toxic activity from Trypanosoma congolense, Experientia 32 (1976) 1533-1534.

[36] Tizard I.R., Holmes W.L., York D.A., Mellors A., The generation and identification of the hemolysin of Trypanosoma congolense, Experientia 33 (1977) 901-902.

[37] Tizard I.R., Sheppard J., Nielsen K., The characterization of a second class of hemolysins of Trypanosoma brucei, Trans. R. Soc. Trop. Med. Hyg. 72 (1978) 198-200. 